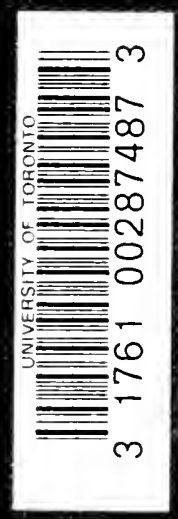






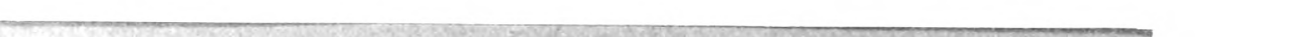




\section{Die jüdischen}

\section{Wanderbewegungen in der neuesten Zeit (1880-1914)}

\section{Inaugural-Dissertation}

Z 111

Erlangung der Würde eines doctor rerum politicarum der Rechts- und Staatswissenschaftlichen Fakultät der Schlesischen Friedrich-Wilhelms-Universität zu Breslau

\section{Wladimir Wolf Kaplun-Kogan}

aus Jalta (Südrussland)

Bonn a. Rh. 1919

A. $M$ a rcus \& E. W e bers $V \in r l$ ag
(Dr. Altert Ahn) 


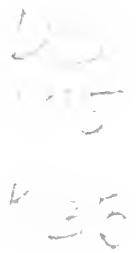

Gednuckt mit Genehmigung der Rechts- Ind Staatswissenschaftlichen Fakultät der Schlesischen Friedrich-Wihnelnus-Universität zu Breslau Berichterstatter: Professor Dr. Adolf Weber

Mit Genemnigung der hohen Rechls- Ind Staatswissenschaftlichen Fakultät wird ein Teildruck der Gesantarbeit vorgelegt. Diese erscheint unter Aem gleichen Titel in diesem Sommer in A. Marcus \&. E. Webers l'erlag in Boml1. 


\section{Lebenslauf}

Ich, Wladimir Wolf Kaplun-Kogan, wurde am 3. März 1888 als Sohn des Großkaufmanns Paul KaplunKogan und seiner Gattin Ljubow, geb. Pechowitsch in Jalta (Südrußland) geboren. Ich bin russischer Staatsangehöriger und mosaischer Konfession. Nachdem ich am humanistischen Gymnasium in Kertsch die Reifeprüfung im Jahre 1909 bestanclen habe, studierte ich drei Semester Jurisprudenz und Volkswirtschaftslehre an der Universität Bonn am Rhein, ging dann zur Handelshochschule über und bestand im Jahre 1913 die Diplomprüfung an der Handelshochschule in Köln a. Rh. Ich ging daraufhin nach Rußland, um das russische Bankwesen zu studieren und arbeitete dort in den Filialen der Azow-Donschen Kommerzbank in Jalta, Theodossia und Mariupol. Kurz vor Ausbruch des Krieges kehrte ich nach Deutschland zurück und war seit Ausbruch des Krieges als wissenschaftlicher Hilfsarbeiter im Komitee für den Osten in Berlin tätig. In Sommersemester 1918 wurde ich an der Berliner Universität immatrikuliert und studierte dort Volkswirtschaftslehre. Seit November 1918 bin ich wirtschaftlicher Beirat am OsteuropaInstitut in Breslau. Meine Lehrer sind: Prof. Dr. Adolf Weber (Breslau) und Prof. Dr. Werner Sombart (Berlin). 


$$
\text { • }
$$


Erstes Kapitel

Die jüdischen Wanderungen in der jüdischen Wissenschaft

\section{Allgemeines}

Das Bestreben, die jüdischen Wanderbewegungen wissenschaftlich $\mathrm{zu}$ erforschen und ihre Ursachen klarzulegen, ist verhältnismäßig neuen Datums. Obwohl das jüdische Volk seit der Zerstörung des zweiten Tempels sich in einer ununterbrochenen Bewegung befindet - und auch schon früher eine Reihe von bedeutenden Wanderungen durchgemacht hat - empfand es bis vor kurzer Zeit diesen Wanderungsprozeß nicht als etwas Anormales. Die ganze Zeit bis zum Ausgang des 18. Jahrhunderts wurde das Phänomen Wanderung rein religiös bewertet: als Sühne, als Mission, als indirekter Weg nach Palästina - je nach der Art der religiös-geistigen Anschauung, die im gegebenen Augenblick das Judentum beherrschte. Und nun vollends im 19. Jahrhundert, in dem die Befreiung der Judenheit vom Ghetto proklamiert wurde, konnte alles andere als eine Konzentration des jüdischen Interesses auf das Wanderungsproblem erwartet und entdeckt werden. Denn es war das Jahrhundert der groß angelegten und vielleicht der erfolgreichsten Assimilationen, die Zeit der Seßhaftmachung des jüdischen Volkes in Europa. Man war froh in dem ungewohnten Gefühl der neu erworbenen, anscheinend festen und echten Staatszugehörigkeit. Um so bitterer wirkte die Enttäuschung, als im letzten Viertel des 19. Jahrhunderts das jüdische Volk wieder auf die Wanderung gehen mußte. Die alten größeren Zentren schienen in einer 
Auflösung begriffen zu sein, die kleineren wurden durch die Zuwanderer in ihrer Festigkeit bedroht - und nete entstanden: mächtiger, anders aufgebaut, mit einer eigen- und fremdartigen ökonomischen und rechtlichen Struktur.

Die ersten Versuche, den Wanderungsprozeß zu.erfassen, wurden alle von dem Gedanken getragen, der Bewegung möglichst schnell und sicher Herr zu werden. Dieses anfängliche Bestreben ging dahin, unter Berücksichtigung der vorhandenen Wanderbewegungen und durch Entfachung der religiösen Leidenschaften des Volkes diesen Wanderungen eine Richtung zu geben, die ihnen ein Ende bereiten würde. Von diesem Gesichtspunkte aus sind alle falsche Messias des Mittelalters zu versiehen. In der neuen Zeit entkleidete man das Bestreben der religiösen Hülle, der utopische Gedanke blieb jedoch auch den neueren Plänen anhaften. Ich denke hier vor allem, um nur ein krasses Beispiel zu geben, an die Ausführungen von Theodor Herzl, der ja bis zum Jahre 1923 die Übersiedlung eines großen Teils des jüdischen Volkes nach Palästina plante. Die Frage, die sich die zahlreichen geistigen Vorgänger $H$ erzls ${ }^{3}$ ) - auf die ich hier näher nicht eingehen kann - und seine ersten Mitarbeiter vorlegten, war nicht die nach dem Wesen der jüdischen Wanderbewegungen, sondern ausschließlich die nach der Verwertung und dem Ziel und dem Ende der jüdischen Emigration. Diese Epoche, die ich als die Zeit des

1) Eine Geschichte der jüdischen messianischen Ecwegung im Mittelalter gibt es nicht. Es kommen also zunächst die betreffenden Stellen aus allgemeinen Geschichtswerken in Betracht. So bei Graetz: Geschichte des jüdischen Volkes, Leipzig 1900, Bd. 8, und S. D ub now: Geschichte des jüdischen Volkes, St. Petersburg 1910, Bd. 3 (russisch). Die Schriften Herzls sind gesammelt in: Dr. Theodor Herzls zionistische Schriften, zwei Teile, Berlin-Charlottenburg. In diesem Zusammenhang sei noch die Biographie von $\mathrm{H}$ erzl von Dr. Adolf Friedema nn: Das Leben Theodor Herzls, Berlin 1914. erwähnt, in der gerade das Messianische der Herzlschen Sendung herausgearbeitet ist, und $\mathrm{A} c \mathrm{~h}$ a d $\mathrm{H}$ a a $\mathrm{m}$ : Am Scheidewege, Essays, Bd. 2, Berlin 1916, der das Utopische der Bewegung eindringlich vor Augen fuihrt. 
Dilettantismus und des Utopismus bezeichnen möchte, und die übrigens recht viele gemeinsame Züge mit der geistigen Struktur des utopischen Sozialismus aufweist, wurde am Anfang des 20. Jahrhunderts abgelöst durch junge jüdische Wissenschaftler aus den Reihen der jüdischen Arbeiterparteien, die es unternahmen, zunächst in Zeitschriftenaufsätzen das Problem wissenschaftlich und vom ökonomischen Gesichtspunkte aus zı ergründen und zı erfassen.

\section{Literaturnachweis}

Ein Literaturnachweis über die jüdischen Wanderungen hat mit diesen Aufsätzen anzufangen. Ich erwähne hier als den bedeutungsvollen eine größere russische Abhandlung Zwi Awraami's: Jüdische Wanderungsprobleme, Zeitschrift Jüdisches Leben, Heft 9, Jahrgang 1905. Er schrieb damals: „Der Inhalt, die Tendenz, die Beweggründe und sogar die unmittelbaren Ursachen der Wanderbewegungen der Juden bleiben nach wie vor in ihrem ganzen Umfange eine terra incognita für uns . . . insoweit die Emigration an und für sich ein inneres Problem des jüdischen Lebens ist, darüber hat man bei uns nốch nicht einmal nachgedacht... das Emigrationsproblem ist nicht nur nicht gelöst, es ist noch gar nicht verstanden." $\mathrm{Z}$ wi A wra a mi unternahm dann den Versuch, eine Philosophie der jüdischen Wanderbewegungen zu konstruieren. Überaus instruktiv ist die Arbeit von Janowski: Das wandernde Israel in derselben Zeitschrift Jüdisches Leben, Heft 1, Jahrgang 1906. Deutsch ist zuerst 1902 ein beachtenswerter Beitrag zur Wanderungsfrage in der Zeitschrift Ost und West, zweiter Jahrgang, p. 382: Die Emigration im Golus von A lexander $\mathrm{H}$ a us mann erschienen ").

2) Über rechtliche und organisatorische Emigrationsfragen schrieb Dr. B. Kahn: Die jüdische Auswanderung, Ost und West, Berlin 1905, p. 458, und Dr. Th. Vogelste in: Auf Ellis Island, ibid. p. 479. Einzelne wertvolle statistische Angaben enthalten die Jahrgänge der Zeitschrift für Demographie und Statistik der Juden, Berlin (seit 1905). 
Im Jahre 1907 erschien die erste wissenschaftliche Untersuchung über die jüdische Einwanderung nach Amerika von Dr. Karl Vornberg: Jüdische Emigration, Versuch einer statistischen Untersuchung, Kiew 1908, russisch, die die wichtigsten Probleme in mustergültiger Weise aufwarf. Das statistische Material wurde zwar nicht ausgiebig, aber, soweit überhaupt herangezogen, sehr gründlich verarbeitet. Die Arbeit des Russen S. Patkanow: Resultate der Immigrationsstatistik in den Vereinigten Staaten von Amerika aus Rußland im Jahrzehnt 1900 bis 1909, St. Petersburg 1911, russisch, behandelt zwar die gesamte Auswanderung aus Rußland, enthält jedoch recht viele treffende Bemerkungen auch ïber die jüdische Auswanderung.

Und nun begann man in verschiedenen Ländern sich für das Problem zu interessieren. Im Jahre 1912 schrieb Dr. N. Katzenels oh n eine kleine Broschüre unter dem Titel: Die jüdische Wanderung, Berlin. Im Laufe eines Jahres - 1913 - erschienen drei größere Untersuchungen über denselben Gegenstand, ohne daß die Verfasser das geringste voneinander wußten: im März 1913 eine Arbeit von mir: Die Wanderbewegung der Juden, Bonn, deutsch; etwa im September 1913 von Professor Dr. L. Hersch: Le Juif Errant d'aujourdhui. Etude sur l'emigration des israelite de l'Europe orientale aux Etats-unis de l'amerique du Nord, Paris, französisch, und Anfang 1914 von Dr. Jose ph: Jewish Emigration, New-York, englisch. Während ich die gesamten jüdischen Wanderungen seit den biblischen Zeiten bis in die Gegenwart hinein zu schildern versuchte, beschränkten sich Professor Dr. Hersch und Dr. Jos e ph auf die Wanderungen der neuesten Zeit. Die Arbeit von $\mathrm{Hersch}$ ist deshalb besonders wertvoll, weil in ihr u. a. auch die politische Seite des Problems streng wissenschaftlich bearbeitet und Österreich und Rußland recht ausgiebig berücksichtigt worden sind. Die Arbeit von Dr. Jose ph ist mir nur nach einer Besprechung bekannt; infolge des Krieges konnte ich kein Exemplar von Amerika herüber bekommen.

Mit diesen Untersuchungen war jedoch die in Gang 
gekommene Forschungsarbeit nicht abgeschlossen. 1917 gai $\mathrm{D}$ a vis Triets ch eine Sammlung von Aufsätzen: Jüdische Emigration und Kolonisation, Berlin, heraus, in der er eine Reihe von wichtigen Emigrationsfragen bespricht. 1918 erschien eine kleine französische Arbeit von Dr. Ka tze nellenbogen: L'emigration juif, Brüssel, in der jedoch neue Gesichtspunkte nicht zu finden sind. Amtliches und erschöpfendes Material über die Einwanderung nach Amerika enthalten die bekannten Annual reports of the commissioner general of immigration to the secretary of commerce and labor, Washington. 
Zweites Kapitel

\section{Die jüdische Einwanderung in die Vereinigten Staaten von Amerika}

\section{1. Übersicht der Gesamtwanderungen}

Die Wege der jüdischen Wanderer sind mannigfaltig.

Nach absolut zuverlässigen und genauen Angaben der schon erwähnten Annual reports und der sonstigen ähnlichen amtlichen Berichte Englands und Rußlands, und nach den nur annähernden Schätzungen jüdischer Statistiker sind, wie aus der Tabelle I ersichtlich ist, alle Lünder, wo die Juden wohnen, in den Wanderungsprozeß einbezogen. Der Osten Europas bildet das große Reservoir der Auswanderung (2581000 Auswanderer). Das größte Einwanderungsland ist Amerika. Wie sich das Verhältnis der verschiedenen Länder untereinander nach der Zahl der Ein- und Auswanderer stellt, werden wir im nächsten Kapitel kurz zu untersuchen haben.

Das Gesamtergebnis ist imponierend genug: bei einer Gesamtjudenheit von höchstens 12 Millionen haben im letzten Menschenalter weit über 3 Millionen Juden, genau 3055000 Personen, ihr Heimatsland gewechselt. Zwei neue jüdische Zentren entstanden, beide von schwerwiegendem Einfluß auf das jüdische und allgemeine wirtschaftliche und politische Leben, in Amerika und Palästina. 


\section{$-7-$ \\ Tabelle l}

Gesamtübersicht der jüdischen Ein- und Auswanderung

In der Zeit von 1880 bis 1914 wanderten Juden

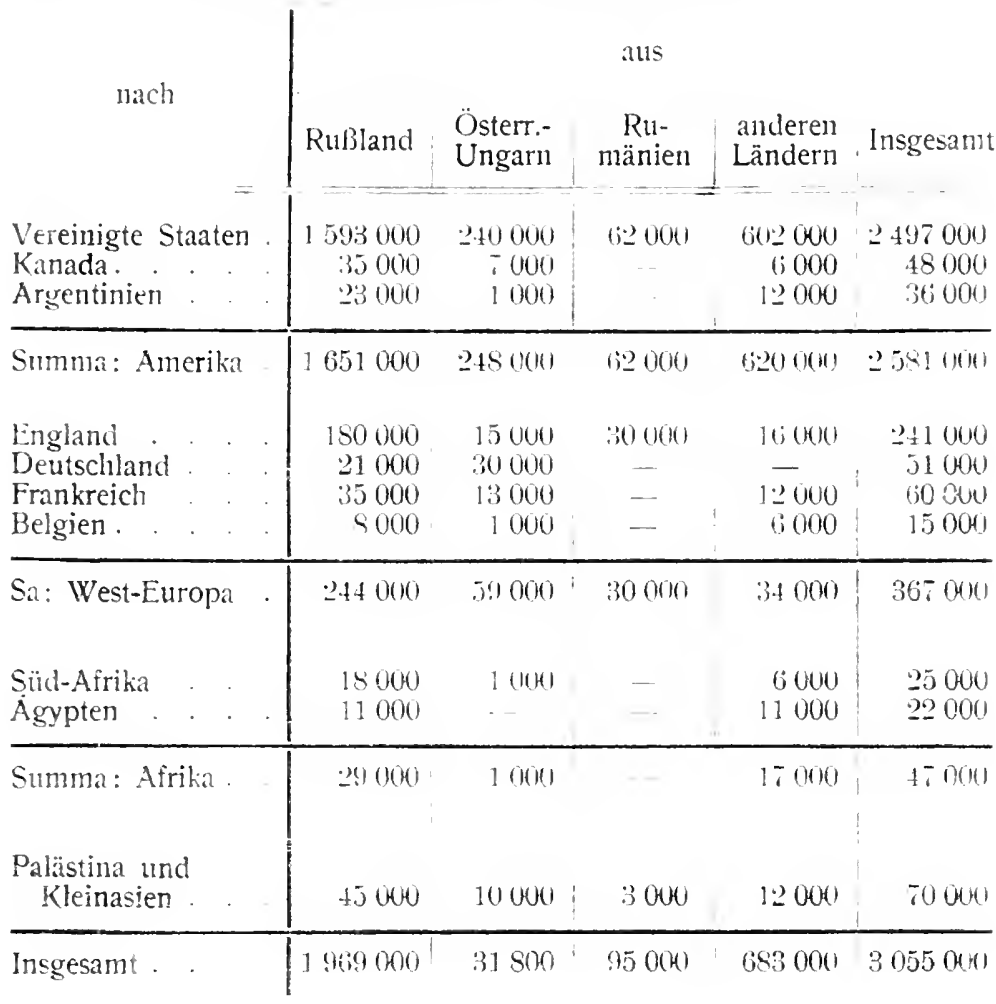

2. Allgemeine und jüdische Einwanderung in die Vereinigten Staaten von Amerika

In folgendem wollen wir die jüdische Einwanderung in die Vereinigten Staaten von Amerika einer genauen Ana. lyse unterziehen. $\mathrm{Da}$ diese Einwanderung numerisch die größte ist - von den 3055000 jüdischen Wanderern der letzten 34 Jahre sind in den Vereinigten Staaten allein 
T abelle II

Jïdische und allgemeine Einwanderung in die Vereinigten
Staaten von Amerika

\begin{tabular}{|c|c|c|c|c|c|c|c|c|c|}
\hline & & & Jïdische & Einus & . & alls & & & \\
\hline $\begin{array}{l}\text { Im Fiskal. } \\
\text { jalıre } \\
\text { (1. Juli bis } \\
\text { 30. Juni) }\end{array}$ & $\begin{array}{l}\text { All- } \\
\text { genteine }\end{array}$ & $\underset{\Xi}{\stackrel{\Xi}{\Xi}}$ & 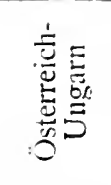 & 它离. & : & & 离莺 & 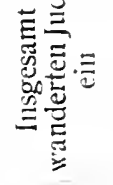 & $\begin{array}{l}\dot{N} \\
= \\
\equiv\end{array}$ \\
\hline $1880 \quad 1881$ & $669+431$ & 8193 & - & & - & - & - & 8193 & 1,2 \\
\hline 11852 & & 17497 & - & - & -- & - & 14310 & 31807 & 4,2 \\
\hline $185: 1883$ & 6013 & 6907 & .. & $\ldots$ & - & $\cdots$ & - & 69.907 & 1,2 \\
\hline $1883158 t$ & .518592 & 15122 & & $\cdots$ & & & 12.285 & $27+110$ & 5,3 \\
\hline $1984 / 1885$ & 395346 & 16603 & - & 一 & -. & - & 19611 & 36,214 & $\because ! 1)$ \\
\hline $5 / 1 S 56$ & 334203 & 17309 & - & & - & - & 29658 & 169967 & 14,0 \\
\hline 61887 & 490109 & 28944 & - & & - & - & 27468 & 56.112 & 11,5 \\
\hline 888 & & .31256 & - & & - & - & 31363 & $6261 !$ & 11,5 \\
\hline $1985 / 1889$ & 4. & 31859 & & - & - & - & 23962 & 55851 & 12,6 \\
\hline 1889,1890 & & $331+\bar{i}$ & & & - & - & $3+303$ & $67+50$ & 14,8 \\
\hline 101891 & & +2145 & -.. & -- & - & - & 69139 & 111284 & $20,1)$ \\
\hline $1 / 189 \%$ & & 9417 & -- & $\ldots$ & .. & - & 0325 & 136742 & 16 \\
\hline $2 / 1893$ & & 626 & - & & - & - & $329+3$ & 68569 & $1.5,1$ \\
\hline 31894 & 28 & ;725 & - & - & - & - & 22105 & 58638 & 20,1 \\
\hline 1894,1895 & 258536 & 33232 & - & 一 & & - & 32077 & 65300 & 26,1 \\
\hline $5 / 1896$ & .343267 & 5137 & & - & - & & 28118 & 73255 & 21,4 \\
\hline 19961897 & & 22750 & $\ldots$ & & - & & 201 & 43434 & 15.11 \\
\hline 18971895 & 229299 & 27221 & - & & $\cdots$ & - & 27409 & $.5+630$ & 24,0 \\
\hline $1895 / 1899$ & 311715 & 34275 & 11071 & 24 & 1343 & 405 & 297 & $37+15$ & 12,11 \\
\hline $1899 / 1900$ & 118 & 37011 & 16920 & 13 & 6183 & 337 & 300 & 60764 & $13,-1$ \\
\hline $1900 / 1901$ & 487915 & 37660 & 13006 & 52 & & 272 & 251 & 58098 & 12,5 \\
\hline $1901 \quad 190^{\circ}$ & $14+8743$ & 37846 & $128+5$ & 55 & 658 & 182 & 169 & 57688 & 4,7 \\
\hline $1902 / 1903$ & 8570.46 & +7689 & 18759 & 420 & $\$ 569$ & 47 & 396 & 76203 & $\rightarrow$ \\
\hline $1: 103 / 190.4$ & & 7544 & 20211 & 817 & $6+46 i$ & 665 & 549 & 106236 & 13,0 \\
\hline 19041905 & 1026499 & 2385 & 17352 & $1+299$ & 38 & 734 & 1283 & 129910 & 12,6 \\
\hline 19051906 & 1100735 & $12523 t$ & 1.48 & 6113 & 38 & 97 & 2666 & $153 \cdot+\mathrm{s}$ & 14,0 \\
\hline 1906,1907 & 128 & 114932 & 185 & 7032 & 3605 & $73 t$ & 3991 & $1+4) 182$ & 11,6 \\
\hline 1907,1900 & 782860 & 71978 & 15293 & 6260 & $4+55$ & 869 & +532 & 103387 & $16,6^{\circ}$ \\
\hline $1908 ; 1909$ & 751785 & 39150 & $8+31$ & 3385 & 1330 & 652 & +543 & 57551 & $7, i$ \\
\hline $1909 / 1910$ & 1041570 & 59824 & 13142 & 4098 & 1701 & 705 & +790 & $8+260$ & 8,0 \\
\hline $1910 / 1911$ & & $65+72$ & 12785 & & 2188 & 799 & $508+$ & 91223 & 10,3 \\
\hline 19111912 & & 58389 & 10757 & & & 629 & & 60595 & 4,6 \\
\hline & 119 & $7+033$ & & & & 80 & & 101330 & 5,1 \\
\hline $1913 / 1914$ & 1218480 & 102638 & 20454 & 3614 & 2646 & $112 \pi$ & 7572 & 138051 & $11,2 i$ \\
\hline
\end{tabular}


2497000 Personen, also 81,7 Proz. der Gesamteinwanderung, angelangt - ist ihr Wesen und ihre Struktur kennzeichnend für alle anderen jüdischen Wanderungen.

Ein Bild der jüdischen und der allgemeinen Einwanderung in die Vereinigten Staaten von Amerikt gibt uns Tabelle II, nach der die jüdische Einwanderung im Durchschnitt 11,42 Proz, ausmacht"). Der prozentuale Anteil der Juden an der Gesamteinwanderung ist in den verschiedenein Jahren ziemlich schwankend, besonders stark war er im Jahre 1894-95: 26,1 Proz.; die Anfang der 90er Jahre erfolgte Austreibung der Juden aus Moskau und den Dörfern dürfte wohl die Ursache der erhöhten Auswanderung bilden. Absolut war die jüdische Auswanderung am stärksten im Jahre 1905-06, in dem 153748 Juden auswanderten (Revolution in Rußland!).

Aus den Städten kommend, wenden sich die Juden auch den Städten zu. So ging über die Hälfte aller neuangekommenen Juden nach Neuyork, im Jahre 1911-12

") Vom Jahre 1908-09 ab werden die Einwohner unterschieden im lmmigrant aliens und nonimmigrant aliens (Tabelle III). Letztere sind zwar auch Fremde, die aber nicht in die Gruppe der Einwanderer eingereiht werden. Die Zahl solcher jüdischer Fremden, deren materielle und kulturelle Lage viel besser ist als die der übrigen Einwanderer, ist minimal.

Tabelle III.

Die Zahl von Nonimmigrant aliens in der gesamten und jüdischen Einwanderung in die Vereinigten Staaten

\begin{tabular}{c|c|c}
\hline Jahr & $\begin{array}{c}\text { Gesamte } \\
\text { Einwanderung }\end{array}$ & $\begin{array}{c}\text { Jüdische } \\
\text { Einwanderung }\end{array}$ \\
\hline $1908-1909$ & 192449 & 3188 \\
$1909-1910$ & 156467 & 3503 \\
$1910-1911$ & 151713 & 3333 \\
$1911-1912$ & 178983 & 3407 \\
$1912-1913$ & 999335 & 4496 \\
$1913-1914$ & 194601 & 4825
\end{tabular}


45111 Personen, d. h. 56,3 Proz. aller Einwanderer, im Jahre 1912-13 50 029, d. h. 58,2 Proz., und im Jahre 1913-14 78575, d. h. 56,9 Proz. Der Rest verteilte sich auf andere amerikanische Großstädte, und nur ein geringer Prozentsatz von etwa 1 bis 2 Proz. siedelte sich auf dem Lande ein.

Überaus lehrreich sind die Zahlen der Tabelle IV (siehe nächste Seite) : unter 39 verschiedenen Nationalitäten, die in den letzten 16 Jahren (1899-1914) eine Wanderarmee von über 13 Millionen aufzuweisen hatten, steht das jüdische Volk mit 1485641 Personen an $z w$ eiter Stelle, was 10,6 Proz. dieser auch in diesen Zeiten ungeheuren Menschenmassen bildet. Süditaliener eröffnen den Reigen (2 690626 Personen, d. h. 19,2 Proz.); an dritter Stelle stehen die Polen (1 402695 Personen, d. h. 10,0 Proz., dann folgen die Deutschen mit einem verhältnismäßig starken Antei! von 7,5 Proz. (1 046925 Einwanderer) ${ }^{4}$ ), nach den Deutschen die Skandinavier (738556 Personen, d. h. 5,4 Proz.), dann die Engländer (622 829 Personen, d. h. 4,4 Proz.), die Irländer (584 813 Personen, d. h. 4,1 Proz.), die Norditaliener (516 759 Personen, d. h. 3,7 Proz.) usw.

\section{Jüdische Einwanderung aus Rußland und Rumänien}

Die jüdische Einwanderung aus Rußland, die bis zum Beginn des 20. Jahrhunderts im Durchschnitt die Hälfte der gesamten jüdischen Einwanderung nach Amerika ausmachte, ist im 20. Jahrhundert noch stärker geworden. Thre Regelmäßigkeit fällt vor allem auf (Tabelle V). Denn wenn wir von den Jahren 1897-99 absehen, in denen auch die allgemeine Einwanderung bedeutend sank, und die gewaltig

$\left.{ }^{4}\right)$ Dieser starke Anteil der deutschen Einwanderer ist dadurch zu erklären, daß unter den Deutschen nicht nur Reichsdeutsche, sondern deutsche Einwanderer überhaupt, aus allen Ländern enthalten sind. So wanderten im Jahre 1913-14 ein: aus Deutschland 29027 Deutsche, d. h. 36,3 Proz. der gesamten deutschen Einwanderung, aus Osterreich-Ungarn 28908 Personen, d. h. 36,2 Proz., aus RuBland 9889, d. h. 12,3 Proz., aus der Schweiz 3039 Deutsche, d. h. 3,8 Proz. 
Tabelle IV

Die Einwanderung in die Vereinigten Staaten in den Jahren 1899-1914 (nach Nationalitäten)

\begin{tabular}{|c|c|c|c|c|c|c|c|c|c|c|c|}
\hline \multicolumn{10}{|c|}{ Nationalität } & \multicolumn{2}{|c|}{$\begin{array}{c}\text { Zahl der Einwandere } \\
\text { absolut in Pro }\end{array}$} \\
\hline 1. Süditalicner & . & & & & & & . & & & 2690626 & $19 \%$ \\
\hline ¿. Juden. & . & . & . & & . & . & . & . & . & 14856.41 & 10,6 \\
\hline$\therefore$ Polen. & . & . & . & & . & . & . & . & . & 1402695 & 10,0 \\
\hline 4. Deutsche & $\therefore$ & $\cdot \dot{x}$ & · & & . & 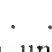 & i & & . & 1046925 & 7,5 \\
\hline Schweden) & 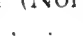 &. & & & . & 111 & & & & 735556 & 54 \\
\hline ti. Engländer & . & . & & & . & & & & . & 622890 & 4,4 \\
\hline ‥ Irländer . & . & . . & . & & . & & & & . & 584813 & 4,1 \\
\hline 4. Norditaliene & $r$ & . . & & & & & & & . & 516759 & 3,7 \\
\hline 1. Slowaken . & & . . & . & & & & & & & 477276 & 3,4 \\
\hline 10. Kroaten lind & Slow & renen & & 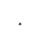 & . & & & . & & 458674 & 3,2 \\
\hline 11. Ungarn . & . &. & . & & . & . & & & . & 456844 & 3,2 \\
\hline 1:2. Griechen . & & . . & . & & . & . & . & . & . & 370074 & 2,6 \\
\hline 1:i. Ruthenen. &.$\quad$. & . . & . & & . & . & . & . & . & $25437 !$ & 1,7 \\
\hline 14. Litaner. & $. \quad-$ & . . & & . & . & . & & . & . & 252594 & 1,7 \\
\hline 1.\%. Schotten. & . . & . & . & & . & . & . & . & . & 223050 & 1,6 \\
\hline 11i. Russen. . & . & . & . & & . & . & & . & . & 221284 & $i, i$ \\
\hline 17. Finnländer & . & . & . & & . & & & & & 193755 & 1,3 \\
\hline 1s. Franzosen & . & . & & & & . & & . & . & 191115 & 1,3 \\
\hline 1!. Japaner . & . . & . . & . & . & . & . . & & . & . & 176719 & 1,2 \\
\hline 20. Bulgaren, Se & erben & und & Mo & ont & enes & grin & & & . & 142441 & 1,0 \\
\hline 21. Holländer 4 & nd $\mathrm{Fl}$ & ämen & & & & & & & & 139526 & 1,0 \\
\hline (2). Böhmen un & d Mäh & iren & (Ts & che & che & & & & & 138870 & 1,0 \\
\hline 23. Rumänen. & 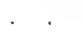 & . . & . & & . & . . & . & . & & 133865 & 0,9 \\
\hline 24. Portugiesen & . & . & . & & . & . & . & . & . & 112982 & 0,7 \\
\hline 25. Mexikaner & . & . & . & & . & . & . & . & . & $10674: 3$ & 0,7 \\
\hline 26. Spanier. & . & . & & & . & & & . & & 88295 & 0,6 \\
\hline 27. Syrier. . & . . &.$\quad$. & . & & . & . & & & & 86111 & 0,6 \\
\hline 24. Afrikaner IS & chwar & ze). & . & & . & & & & & 62191 & 0,4 \\
\hline 2!. Cuba . . &.$\quad$. &.$\quad$. & & & . & & & . & & 57918 & 0,4 \\
\hline (3). Armenier. & & . & & & . & & & & & 51950 & 0,3 \\
\hline 31. Dalmatien, & Bosnie & en un & nd 1 & $\mathrm{He}$ & zeg & & in & & & 49437 & 0,3 \\
\hline 3:. Wales. & . . & . & . & . & . & & & & & 30617 & 0,2 \\
\hline 33. Chinesen & . & . & . & . & . & . & & . & . & 29881 & 0,2 \\
\hline 34. Türken . & . $\cdot$ & . $\cdot$ & . & . & . & . & . & . & . & 19916 & 0,1 \\
\hline 35. Westindien & (auBer & - Cub & ba) & . & . & . & . & & . & 16409 & 0,1 \\
\hline 36. Spanisch-Ar & merika & . & . & & 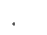 & . & . & & . & 16181 & 0,1 \\
\hline 37. Koreaner . &.$\quad$. & . & . & & . & . & . & & . & 8047 & 0,05 \\
\hline 35. Ostindier. & . & . & . & 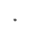 & . & . & . & & & 6828 & 0,04 \\
\hline 3\%. Oceanien. & $\cdot$. & $\cdot \cdot$ & & & . & & & . & . & 1045 & 0,008 \\
\hline Cresamteinwande & rung & . & . & & . & & & & & 13685804 & 100,0 \\
\hline
\end{tabular}




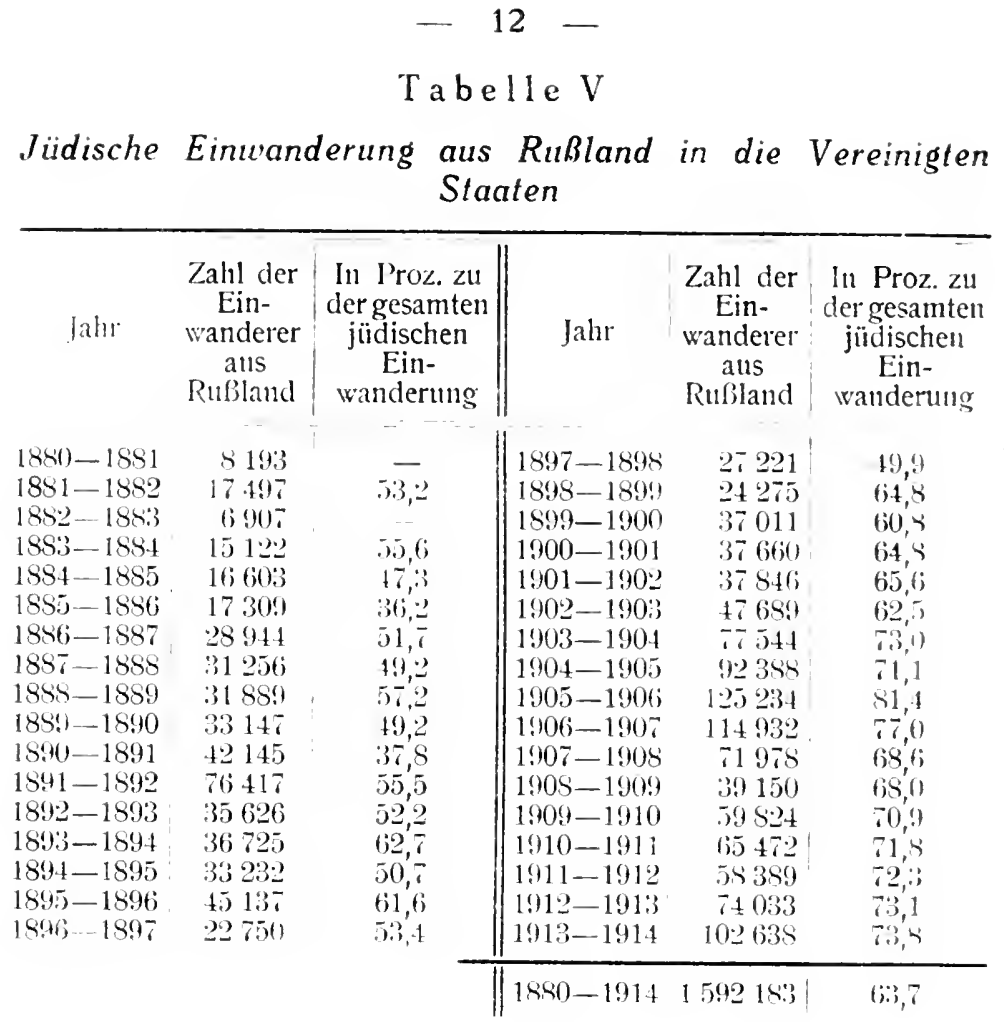

anschwellende Auswanderung in den Jahren 1905-07 aufs Konto der Revolution und Pogrome setzen, so sehen wir, daß die jüdische Auswanderung aus Rußland eine Erscheinung ist, die trotz aller Schwankungen die Tendenz hat, langsam zu steigen. Mögen einzelne Jahre eine niedrigere Zahl der Auswanderer aufweisen, im großen und ganzen ist die jüdische Auswanderung aus Rußland keine sporadische, durch vorübergehende Umstände herbeigeführte, sondern eine systematische, tief im Wesen des jüdischen Lebens in Rußland liegende Erscheinung. Die Tendenz zum Steigen tritt uns noch klarer entgegen, wenn wir nicht einzelne Jahre betrachten, deren Auswandererzahl mancherlei Zufälligkeiten unterworfen ist, sondern größere Zeiträume von fünf Jahren miteinander vergleichen. So ersehen wir 
aus der Tabelle VI, daß im Lustrum 1881 '-85 die jährliche durchschnitliche Einwandererzahl 12865 beträgt; im Lustrum 1886-90 schon mehr als das Doppelte: 28509; im Lustrum 1891-95: 44 829; im Lustrum 1896-1900: 31 278; im Lustrum 1901-05: 58625 und im Lustrum 1906-10: 82 223. Die einzige Ausnahme bildet das Lustrum 1896 bis 1900. In den letzten vier Jahren betrug die durchschnittliche Einwandererzahl aus Rußland 75144 Personen.

$$
\text { Tabelle VI }
$$

Jüdische Einwanderung aus Rußland in die Vereinigten Staten

\begin{tabular}{ccc}
\hline Zeitraum & $\begin{array}{c}\text { Zahl der } \\
\text { Einwanderer }\end{array}$ & $\begin{array}{c}\text { Durchschnittlich } \\
\text { jährlich }\end{array}$ \\
$1881-1885$ & 64322 & 12865 \\
$1886-1890$ & 142545 & 28509 \\
$1891-1895$ & 224145 & 4482. \\
$1896-1900$ & 156394 & 31278 \\
$1901-1905$ & 293127 & .86625 \\
$1906-1910$ & 411138 & $\$ 8223$ \\
$1911--1914$ & 300532 & 75144
\end{tabular}

Das im zweiten Kapitel über das Besondere der Ursachen der jüdischen Auswanderung aus Rußland und Rumänien Ausgeführte wird durch eine Gegenüberstellung der Entwicklung der Auswanderung aus diesen zwei Ländern nur bekräftigt. Wie die Tabelle VII zeigt, ist die jüdische Auswanderung aus Rumänien nicht in einem stetigen Anwachsen begriffen, sondern trotz der vielen Schwankungen in einem ununterbrochenen Sinken: von 11,6 Proz. im Jahre 1900-01 (6827 Auswanderer) ist sie auf 1,2 Proz. im Jahre 1913-14 gesunken (2646 Auswanderer). Aber auch die Schwankungen, denen die Auswanderung unterworfen war, sind hier stärker und unregelmäßiger, als in der Auswanderung aus Rußland. Nicht die Entwicklung des wirtschaftlichen Lebens ist in Rumänien der Grund der Auswanderung, sondern das zufällige, sporadische und unberechenbare Spiel der politischen Kräfte. 


\section{$-14-$ \\ Tabelle VII}

Jüdische Einwanderung aus Rumänien in die Vereiniglen Staaten

\begin{tabular}{|c|c|c|}
\hline Jahr & $\begin{array}{c}\text { Zahl der } \\
\text { Einwanderer } \\
\text { aus Rumänien }\end{array}$ & $\begin{array}{c}\text { In Proz. } \\
\text { zu der gesamten } \\
\text { jüdischen } \\
\text { Einwanderung }\end{array}$ \\
\hline $1898-1899$ & 1343 & 34 \\
\hline $1890-1900$ & $618: 3$ & 10,2 \\
\hline $1900-1901$ & 6527 & 11,6 \\
\hline $1901-1902$ & 6589 & 11,4 \\
\hline $190:-1903$ & 5569 & 11,2 \\
\hline $190: i-1904$ & 6446 & 6,1 \\
\hline $1904-1905$ & 3854 & 2,9 \\
\hline $1905-1906$ & $387 \%$ & 2,5 \\
\hline $1906-1907$ & 3605 & 24 \\
\hline $1907-1904$ & 4.455 & $4, \therefore$ \\
\hline $1908-1909$ & 1330 & $2 ; 3$ \\
\hline $1909-1910$ & 1701 & 2,1 \\
\hline $1910-1911$ & 2188 & $2 ; 3$ \\
\hline $1911-191: 3$ & 1512 & lis \\
\hline $1912-1913$ & 1640 & 1,6 \\
\hline $1413-1914$ & $26+6$ & 1,9 \\
\hline $1899-1914$ & 62913 & 1,2 \\
\hline
\end{tabular}

\section{Geschlechtsaufbau der jüdischen Einwanderung}

Schon aus dem Grade der Beteiligung der jüdischen Fra $u$ an der großen überseeischen Auswanderung können wir auf das hervorragende Merkmal der jüdischen Wanderung der neusten Zeit schließen: Diese Wanderung ist keine vorübergehende, die Einwanderer haben vielmehr den festen Willen, sich im neuen Lande d a u e r n d niederzulassen. Die jüdische Frau, die als die erste auf die Gründung eines Haushaltes drängt und die ihn erst ermöglicht, ist es, die der jüdischen Wanderung ihre Monumentalität verleiht und ihr den Charakter einer Volks- (im Gegensatz zu Klassen-) Wanderung gibt. TabelleVIII unterrichtet über dieVerteilung der jüdischen Wanderer nach dem Geschlecht. In den Jahren 1899-1914 kamen insgesamt 831561 Männer und 654080 Frauen. Unter 100 Einwanderern gab es also 
 \\ Tabelle VIII}

Verteilung der jüdischen Einwanderer nach dem Geschlecht

\begin{tabular}{|c|c|c|c|c|c|}
\hline Jahr & Zusammen & Mämulich & Proz. & Weiblich & Proz. \\
\hline $1895 i--1899$ & 37415 & 2153 & $5 \pi$ & 16262 & $4 ;$ \\
\hline $1899-1900$ & lio itit & 36330 & 60 & 24434 & .10 \\
\hline $1900-19001$ & 58095 & 3234 & $5 t ;$ & 25) 753 & 14 \\
\hline $1901-1902$ & 57698 & 32737 & $5 i$ & $2+951$ & $4: 3$ \\
\hline $1902-1903$ & 76203 & 43985 & $5 n$ & 32218 & 42 \\
\hline $1903-1904$ & 106236 & $650+10$ & $6 i 1$ & 41196 & 39 \\
\hline $190+-1905$ & 199910 & 42076 & $6 i 3$ & 4783.1 & $: 37$ \\
\hline $1905-1906$ & 15374 & $\$ 0086$ & .52 & 73662 & $4 S$ \\
\hline $1906-1907$ & 149182 & $\$ 0530$ & 54 & 68652 & $4 t ;$ \\
\hline $1907-1908$ & $10338 \div$ & .3627. & it & 47110 & 46 \\
\hline $1908-1909$ & 57551 & $3105 \pi$ & 54 & $2649 t$ & 46 \\
\hline $1909-1910$ & 84260 & 46206 & 55 & 38054 & 45 \\
\hline $1910-1911$ & $9122 \%$ & +5935 & 54 & 42285 & 46 \\
\hline $1911-1912$ & $\$ 0595$ & 12751 & 53 & 37844 & 47 \\
\hline $1912-1913$ & 101330 & 57148 & 56 & +4182 & 44 \\
\hline $191: 3-191+$ & 135051 & $7+905$ & i4 & 63146 & 46 \\
\hline $15 ! 99-19+1+$ & $1+55(6+1$ & 831561 & if & $(i n+080)$ & +1 \\
\hline
\end{tabular}

Geschlechtsaufbau der gesamten und der jüdischen Einwanderermasse in den Jahren 1912-1914

\begin{tabular}{|c|c|c|c|c|c|c|}
\hline \multirow[b]{2}{*}{ Jahr } & \multicolumn{3}{|c|}{ Gesamteinwanderung } & \multicolumn{3}{|c|}{ Juden } \\
\hline & Männer & Frauen & $\begin{array}{c}\text { Auf } 1000 \\
\text { Männer } \\
\text { trafen } \\
\text { Frauen }\end{array}$ & Männer & Frauen & $\begin{array}{c}\text { Auf } 10 x) \\
\text { Männer } \\
\text { trafen } \\
\text { Frauent }\end{array}$ \\
\hline $1911-1912$ & 529931 & $30 S 2.41$ & 582 & 42751 & $3784 t$ & 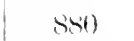 \\
\hline $1912-1913$ & $\operatorname{sos} 14 t$ & 3897.48 & 482 & 57148 & $4+182$ & 775 \\
\hline $1913-1914$ & $79574 \%$ & 119733 & 525 & 74905 & 63146 & 512 \\
\hline $1912-1914$ & 2136822 & 1117722 & $52: 3$ & $17+80 t$ & 145172 & $82 !)$ \\
\hline
\end{tabular}

56 Männern und 44 Frauen. Betrachten wir die einzelnen Jahre für sich, so fällt sogleich die Regelmäßigkeit der Verteilung der beiden Geschlechter auf; das Verhältnis hat sich in den letzten 8 Jahren sozusagen stabilisiert; denn es 


\section{$-16-$ \\ Tabelle $\mathrm{X}$}

Geschlechtsaufbau der verschiedenen Nationalitäten in der Einwanderung im Jahre 1913-1914

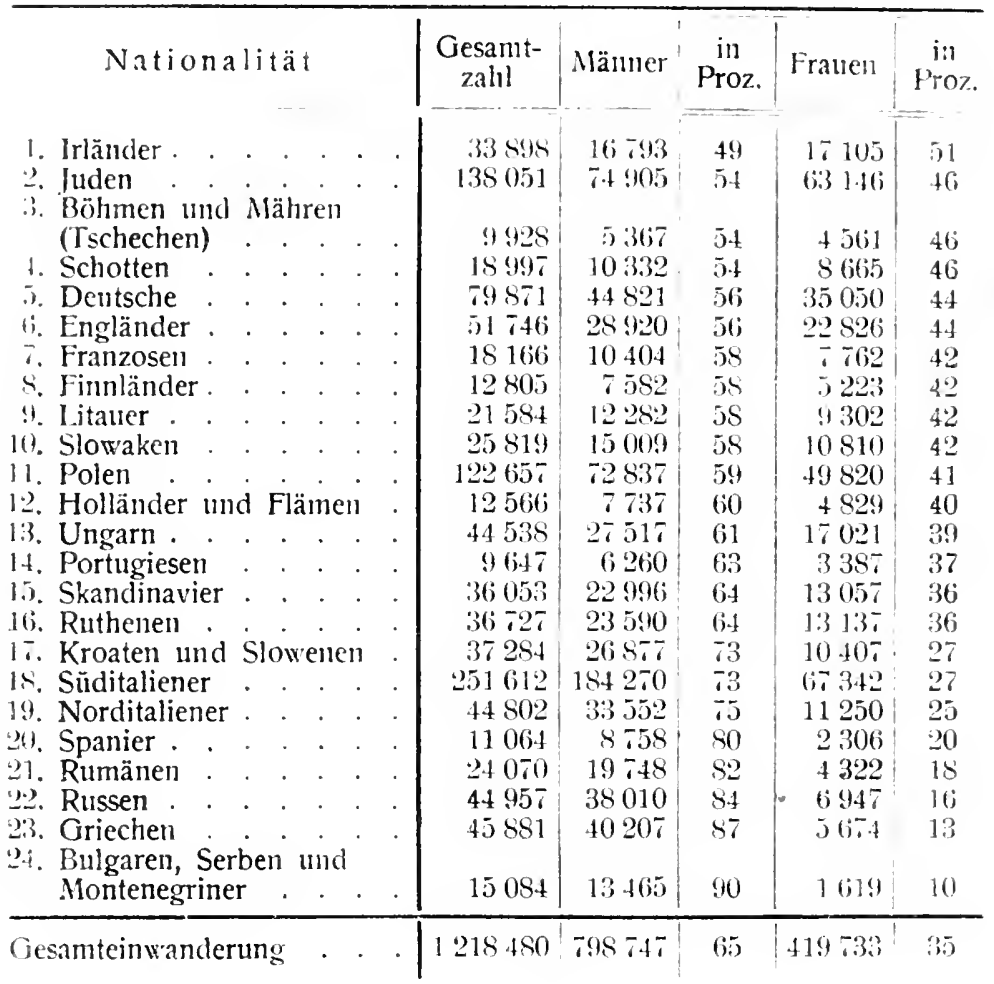

kamen in diesen 8 Jahren fünfmal auf 54 Männer immer 46 Frauen. Die übermäßig starke Beteiligung der Männer in den Jahren 1903-04 (61 Männer und 39 Frauen) und 1904-05 (63 Männer und 37 Frauen) ist durch die Kriegsereignisse dieser Jahre zu erklären.

Vergleichen wir den Geschlechtsaufbau der jüdischen Einwanderer mit demjenigen der Gesamteinwanderung, so tritt uns das Überwiegen der Frauen bei den Juden noch klarer entgegen (Tabelle IX). 
In den letzten 3 Jahren (1912-1914) kamen auf 1000 Männer bei der Gesamteinwanderung 523 Frauen, bei den Juden 829 Frauen. Es ist bezeichnend, daß die Geschlechtsgliederung der jüdischen Bevölkerung in den hauptsächlichsten Auswanderungsländern Rußland und Galizien, wo ja das Überwiegen der Frauen durch stärkere Auswanderung der Männer herbeigeführt wird, sich nicht allzu stark von dem Geschlechtsaufbau in der Auswanderung unterscheidet. So entfielen (1897) auf 1000 Männer.

in den 15 Gouv. des russischen Ansiedlungsrayons

in den 10 Gouv. Polens

3 Ostseeprovinzen

". ". ibrigen 32 Gouv. des Euro-

päischen Rußlands

1061,9 Frauen $1057,1 \quad$ " 1001,2

\section{6,7}

In Galizien und Bukowina entfielen (1910) auf 1000 Männer 1054 Frauen. Unter allen Nationalitäten stehen die Juden nach der Zahl der Frauen in der Einwanderung an zweiter Stelle (allerdings zusammen mit Tschechen und Schotten). An erster Stelle stehen die Irländer, bei denen 51 Männer und 49 Frauen (unter 100 Personen) einwanderten (Tab. X). In der Gesamteinwanderung kamen im Jahre 1913-14 unter 100 Einwanderern 65 Männer und 35 Frauen.

\section{Altersaufbau der jüdischen Einwanderung}

Der Geschlechtsaufbau der Auswanderung weist ein dem Geschlechtsaufbau im Auswanderungslande ähnliches Bild auf. Es wandern nicht einzelne Männer und Frauen allein, sondern es wandert ein in sich geschlossener Teil der Bevölkerung, ein Stück des Volkes aus: Mann, Frau - und $\mathrm{K}$ ind, wie der Altersaufbau zeigt (Tabelle XI). Der Familie n charakter der jüdischen Einwanderung, der daraus zu ersehen ist, macht zwar die jüdische Wanderung zu einer Volkswanderung, gestaltet aber gleichzeitig den wirtschaftlichen Kampf der Neuangekommenen überaus ungünstig: Die große Anzahl der Arbeitsunfähigen erschwert zecht sehr die Lage der verhältnismäßig kleinen Anzahl der Arbeitsfähigen. 
Tabelle XI

Altersaufbau der jïdischen Einwanderer

\begin{tabular}{|c|c|c|c|c|c|c|}
\hline \multirow[b]{2}{*}{ Jalir } & \multicolumn{6}{|c|}{ Altersklasse } \\
\hline & $\begin{array}{l}\text { Inter } \\
\text { 11 Jahren. }\end{array}$ & Proz. & 1.t-4t Jahre & Proz. & $\begin{array}{l}\text { über } \\
4.5 \text { Jahre }\end{array}$ & Proz. \\
\hline $1498-18999$ & 898. & 24 & $26 ; 019$ & (6) & $2+09$ & i \\
\hline $18909-1500)$ & 13092 & 21 & 14239 & $\because: 3$ & 4.33 & ti \\
\hline $1900-19001$ & $1.4 \div 3$ & 85 & 39830 & bis & $35: 37$ & 7 \\
\hline $15(0) 1-19002$ & $15: 212$ & 26 & 38937 & (i) & $: 1399$ & $i$ \\
\hline $1000-19(0) ;$ & 19011 & 25 & $53(1-4$ & .0 & 1085 & 5 \\
\hline $1003-19004$ & $\begin{array}{l}235 \\
-3\end{array}$ & :.) & 7.24 & 3 & $5+53$ & 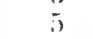 \\
\hline $1904-190$ & i & 2 & 9596.1 & 7.1 & 5393 & 1 \\
\hline $1005-1906$ & $13(i 00)$ & 3 & $1+113.5$ & 66 & +253 & 6 \\
\hline $1906-1907$ & $: ? 6964$ & 2.5 & $1+137.9$ & 71 & $-70 \%$ & i \\
\hline $1907-1904$ & 20013 & 25 & 7138 & (i) & 5156 & $i$ \\
\hline $1905-1909$ & 15210 & 20 & 346 & 67 & 38.6 & $\bar{r}$ \\
\hline $1909-1910$ & $31+60$ & 26 & 57191 & 65 & 5200 & fi \\
\hline $1910-1911$ & 21835 & 24 & $(3) 674$, & in & 5714 & 6 \\
\hline $1911-1912$ & 3000.1 & 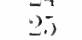 & 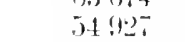 & is & $55 \%$ & 7 \\
\hline $1912-1913$ & 20,3 & 2.3 & 72018 & 71 & $(i)-34$ & $\because$ \\
\hline $1913 \ldots-1 ! 111$ & $3011 \%$ & 2.2 & 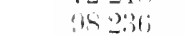 & -1 & -00 & $\frac{1}{1}$ \\
\hline $18 ! 19-1911$ & $3(32017: 3$ & $\because$ & 103010 & $16: 1$ & $4(j) i 20$ & 7 \\
\hline
\end{tabular}

Die amerikanische Statistik unterscheidet drei Altersklassen: 1. unter 14 Jahren, 2. zwischen 14 und 44 Jahren, und 3. über 55 Jahre. Die Zahl der Kinder unter 14 Jahren schwankt bei den Juden zwischen 21 und 28 Proz. und bildet im Durchschnitt in den letzten Jahren 24 Proz. Die Zahl der Erwachsenen (2. Altersklasse) schwankt zwischen 65 und 74 Proz. und bildet im Durchschnitt 69 Proz.; die Zahl der Alten über 45 Jahre macht im Durchschnitt 7 Proz., was dem Prozentsatz in der Gesamleinwanderung gleichkommt; hier bietet die jüdische Einwanderung keine Abweichung von der gesamten und italienischen Einwanderung (Tabelle XII). Die beiden anderen Altersklassen sind hingegen bei der Gesamteinwanderung viel normaler besetzt: "... hier haben wir den spezifischen Altersaufbau der Einwanderungsbevölkerung: schwache Besetzung der jüngsten Altersklassen (1-14 Jahre), gewaltige Ausbauchung bei den produktiven Klassen (14-44 Jahre) und Konvergierung 
in den höheren Altersklassen ... 81 Proz. machen die produktiven Altersklassen aus "). Bei den Juden allein ist es ganz anders, wo $1 / 4$ der Einwanderer sich im Alter von 1-14 Jahren befindet!

Der Familiencharakter der jüdischen Einwanderung, den wir unten noch näher kennen lernen werden, bringt es mit sich, daß die Auswanderung der Juden aus Amerika

$$
\text { Tabelle XII }
$$

Altersaufbau der gesamten, jüdischen und italienischen Einwanderung in Jahre 1913-1914

\begin{tabular}{|c|c|c|c|c|c|c|}
\hline \multirow[b]{2}{*}{ Altersklasse } & \multicolumn{3}{|c|}{ Absolut } & \multicolumn{3}{|c|}{ in Prozent } \\
\hline & $\begin{array}{l}\text { Gesamt- } \\
\text { einwande- } \\
\text { rung }\end{array}$ & Juden & Italiener & $\begin{array}{l}\text { Gesamt- } \\
\text { einwande- } \\
\text { rung }\end{array}$ & Juden & Italiener \\
\hline unter 1 t Jahren & 1.5621 & 30113 & 37 :31 & 12 & $2 \cdot 2$ & $13^{\circ}$ \\
\hline $14-44$ Jalire & 931692 & 15236 & 239534 & $\$ 1$ & 71 & $<1$ \\
\hline ïber 45 Jahre & 78167 & $\because 702$ & 19169 & 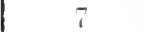 & $T$ & i \\
\hline
\end{tabular}

im Vergleich zu den anderen Nationalitäten minimal ist. Wir werden die jüdische Auswanderung aus Amerika in einem besonderen Kapitel ausführlicher beschreiben. Daß die Juden keine Saisonwanderer sind und von dem Wunsche getragen werden, in der neuen Heimat sich $d$ a u e r nd niederzulassen, beweist die kleine Zahl derjenigen Einwanderer, die schon einmal in Amerika gewesen sind. Die Zahl solcher ",birds of passage" ist bei den Juden verschwindend gering: 1-4 Proz, während bei der Gesamteinwanderung ,birds of passage" 8-21 Proz. ausmachen! (Tab. XIII.)

\section{Familienstand der jüdischen Einwanderung}

Die überaus fein angelegte amerikanische Statistik ermöglicht uns seit der Neuerung vom Jahre 1909-10, in dem.

J) Dr. J. Sega II: Die Einwanderung der Juden in die Vereinigten Staaten im Jahre 1907-08, Zeitschrift für Demographie und Statistik der Juden, 1909, p. 55. 
eine besondere Aufnahme über die Familienverhältnisse der Einwanderer zum ersten Male vorgenommen wurde, auch über den Familienstand der jüdischen Einwanderer ein klares und eindringliches Bild $\mathrm{zu}$ gewinnen. Die Tabellen XIV und XV, welche die einzelnen Jahreszahlen und das

\section{Tabelle XIII}

Prozentsatz der Einwanderer, die in den Vereinigten Staaten schon wenigstens einmal gewesen sind

\begin{tabular}{c|cc} 
Jahr & $\begin{array}{c}\text { Unter allen } \\
\text { Einwanderern }\end{array}$ & $\begin{array}{c}\text { Unter juidischen } \\
\text { Einwanderern }\end{array}$ \\
\hline $1898-1899$ & 18,6 & 4,6 \\
$1899-1900$ & 15,4 & 3,3 \\
$1900-1901$ & 11,6 & 4,3 \\
$1901-1902$ & 5,5 & 2,8 \\
$1902-1903$ & 8,5 & 1,9 \\
$1903-1904$ & 12,8 & 1,9 \\
$1904-1905$ & 17,1 & 2,07 \\
$1905-1906$ & - & 1,73 \\
$1906-1907$ & $-5,17$ \\
$1907-1908$ & 5,57 & 1,17 \\
$1908-1909$ & 21,19 & 4,39
\end{tabular}

Gesamtergebnis der letzten 5 Jahre (1910-14) enthalten, geben über die uns interessierende Frage ausreichende Auskunft. Den Kinderreichtum der jüdischen Einwanderung kennen wir schon. So maclien bei den Juden die Knaben unter 14 Jahren, die ledig sinả, im Lustrum 1910-14 11,9 Proz. Die ledigen Mädchen unter 14 Jahren ") sind etwas schwächer vertreten: 11,5 Proz. Die Struktur der jüdischen Einwanderung, die ein getrcues Bild der Bevölkerungsverhältnisse in den Auswanderungsländern darstellt, ist auch in diesem Punkte überaus bezeichnend: das bekannte Überwiegen der Knaben in der jüdischen Bevölkerung Rußlands spiegelt sich in den oben angegebenen Zahlen der Einwanderung wieder.

4) Als Kuriosum sei erwähnt, daß auch in diesem Alter einige Mädchen schon verheiratet waren. 
Bei der gesamten und italienischen Einwanderung sind die Knaben und Mädchen bedeutend schwächer vertreten: es gibt ihrer dort nur halb so wenig wie bei den Juden.

Viel interessanter ist die zweite Altersklasse: zwischen 14 und 44 Jahren. Was die Ledigen unter ihnen anbetrifft, so gab es bei den Juden weniger ledige mä nnlich e Personen als bei der Gesamteinwanderung, auch weniger als bei den Italienern; es kamen im Lustrum 1910-14 auf je 100 jüdische Einwanderer 25,3 männliche ledige Personen zwischen 14 und 44 Jahren, bei der Gesamteinwanderung 33,2, bei den Italienern 33,4. Dafür gab es bei den Juden mehr ledige we iblich e Personen; so kamen in demselben Jahre auf 100 Juden 18,8 ledige Mädchen im Alter von 14-44 Jahren, bei der Gesamteinwanderung jedoch nur 14,8 , bei den Italienern sogar nur 7,7. Schon daraus allein sieht man klar den Familiencharakter der jüdischen Einwanderung. Während es in der Gesamteinwanderung mehr jüngere freie Männer gibt, die leichter und ungehinderter ihre Arbeitskraft in der neuen Heimat verkaufen können, und die Mädchen schwächer vertreten sind, ist die Anzahl der ledigen Frauen bei den Juden sehr bedeutend; es sind meistens Töchter, die zu den jüdischen Familien gehören. $\mathrm{Da}$ dem wirklich so ist, ersehen wir aus der Tabelle XV, die uns über den Altersaufbau der ledigen Frauen in der ¡üdischen, gesamten und italienischen Einwanderung Auskunft gibt. Demnach gab es unter den ledigen jüdischen Frauen im Alter von 14-21 Jahren im Lustrum 1910-14 14,2 Proz., bei der Gesamteinwanderung nur 9,1, bei den Italienern sogar nur 4,5. Im Alter von 20-29 Jahren gab es unter den Jüdinnen fast ebensoviel ledige Personen wie bei der Gesamteinwanderung (ungefähr 4 Proz.), bedeutend mehr jedoch als bei den Italienern (3,5 Proz. gegen 2,2). Im Gegenteil aber gab es unter den Jüdinnen weniger ledige Personen im Alter von 30 bis 37 Jahren: 0,4 Proz. gegen 1,1 Proz. bei der Gesamteinwanderung. Alle diese Zahlen entrollen dasselbe Bild. Auch unter den ledigen jüdischen weiblichen Personen haben wir mehr junge Mädchen als bei der Gesamteinwanderung und bei den Italienern, weniger dagegen alleinstehende Frauen in dem Alter, in 


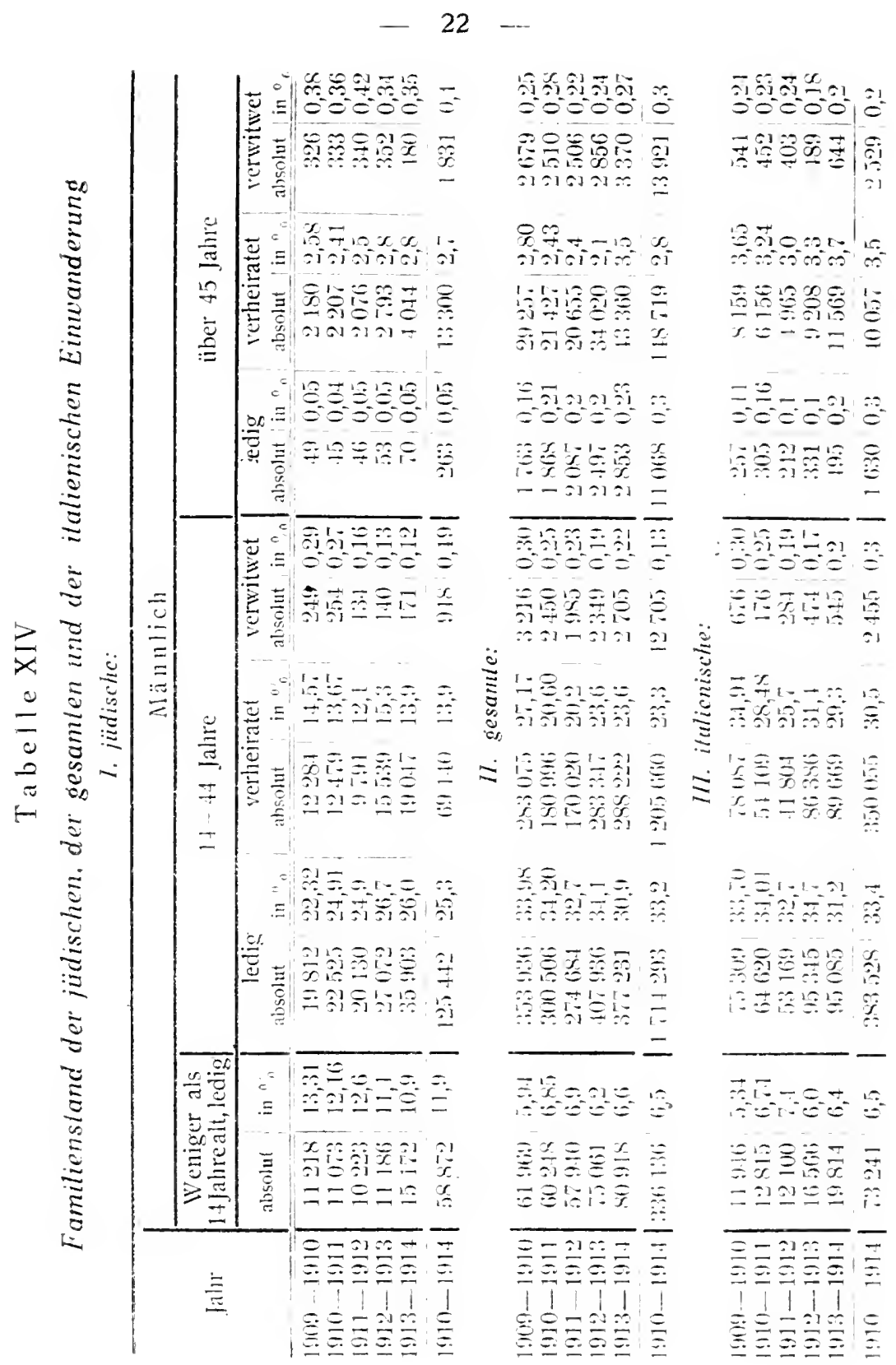




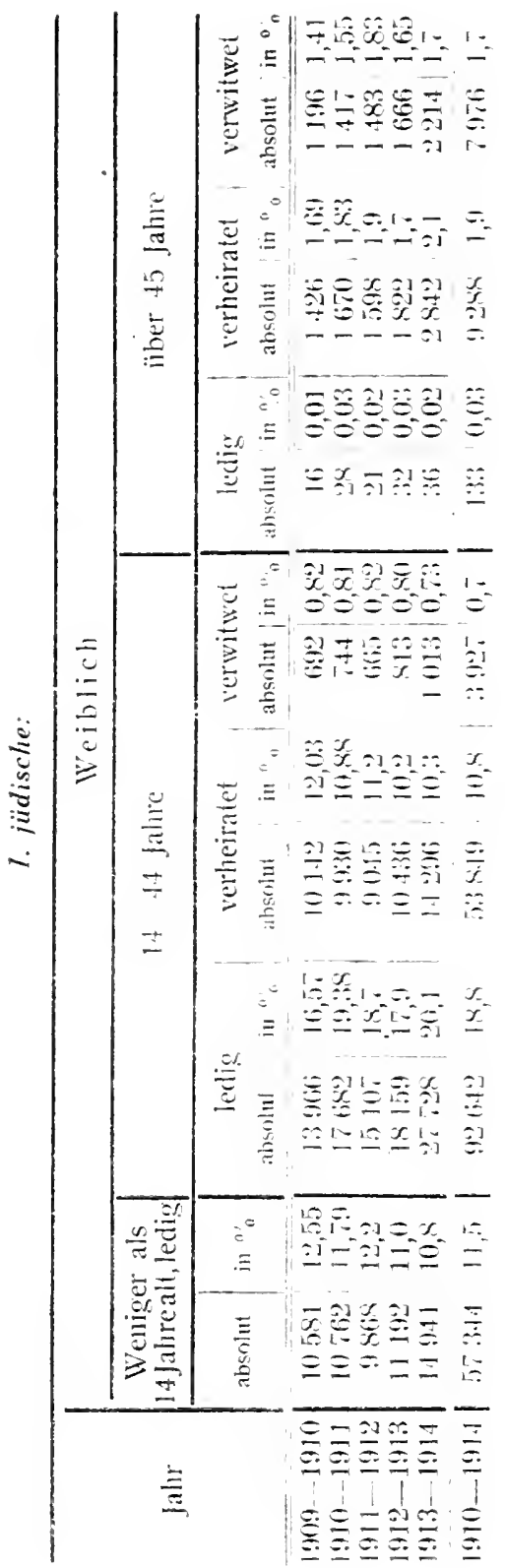

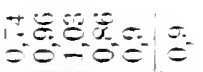

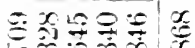

$1-x \times 5=$

$0 \div 0=01=$

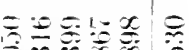

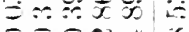

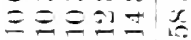

$-1-$

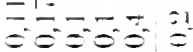

$y=\equiv \div=$

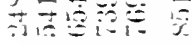

은

$\Rightarrow=05=$

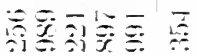

$\because n-1=10$

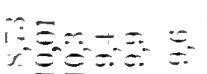

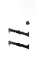

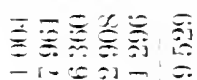

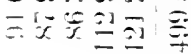

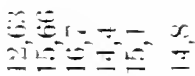

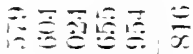

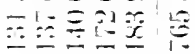

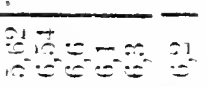

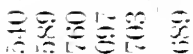

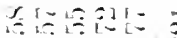

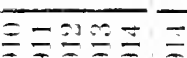

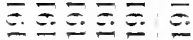

gㄹ.

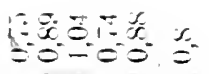

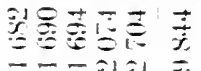

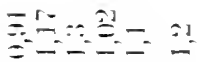

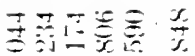

3010100201

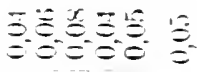

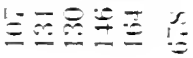

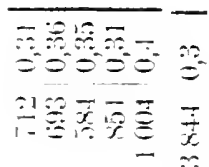

$x=x-10 x$
$x=2=0 x$

二10

$-12=0$

$x=0,05=$

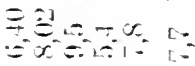

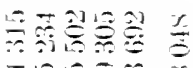

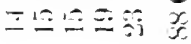

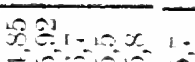

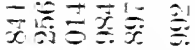
シニニコー

主志家家

氞家家 


\section{$-24^{\circ}-$ \\ Tabelle XV}

Altersaufbau der ledigen Frauen in der jüdischen, gesamten und italienischen Einwanderung in den Jahren 1910-1914

1. jüdische:

\begin{tabular}{|c|c|c|c|c|c|c|c|c|}
\hline \multirow{3}{*}{ Jah.. } & \multicolumn{8}{|c|}{ Es gab unter den ledigen Franen ini Alter von } \\
\hline & \multicolumn{2}{|c|}{$14-21$ Jahren } & \multicolumn{2}{|c|}{$22--29$ Jahren } & \multicolumn{2}{|c|}{30 -37 Jahren } & \multicolumn{2}{|c|}{$34-44$ Jahres } \\
\hline & absolut & $\begin{array}{l}\text { in } \\
\text { Proz. }\end{array}$ & absolut & $\begin{array}{c}\text { itu } \\
\text { Proz. }\end{array}$ & absolut & $\operatorname{in}_{\text {Proz. }}$ & absolut & $\operatorname{in}_{\text {int }}$ \\
\hline $1910-1911$ & 30.341 & 14,2 & 17430 & 3,5 & $204: 3$ & 0,4 & 63 & $0,0 y$ \\
\hline \multicolumn{9}{|c|}{ 11. gesamte: } \\
\hline $1910-1911$ & 170105 & $\because, 1$ & 206561 & 3,9 & $54.2 \cdot 2$ & 1,1 & 16927 & 0,3 \\
\hline \multicolumn{9}{|c|}{ III. italienische: } \\
\hline $1910-1911$ & 520.5 & 1,5 & 25069 & 2,2 & 50.1 & 11,5 & 163.3 & 0,2 \\
\hline
\end{tabular}

dem sie meistens schon verheiratet sind: zwischen 30 und 37 Jahren. Dies ist auch das Alier, in dem sie als Wirtschaftssubjekte für den Daseinskampf vorwiegend in Frage kommen.

Viel klarer stellt die Verhältnisse die Rubrik der Verheiraieten dar. Hier ist es beachienswert, daß die Differenz zwischen dem Prozentsatz der verhcirateten männlichen Personen im Alter von 14-44 Jahren und dem Prozentsatz der verheirateten weiblichen Personen desselben Alters bei den Juden ganz unbedeutend ist, während bei der Gesamteinwanderung und den Italienern, die ja nur vorübergehend nach Amerika übersiedeln, der Prozentsatz der verheiraleten Männer bedeutend den der verheirateten Frauen übersteigt. So gab es im Lustrum 1910-14 bei den Juden 13,9 Proz. der verheirateten männlichen Personen und 10,8 Proz. der verheirateten weiblichen Personen in demselben Alter $(14$ bis 44 Jahre). Die Differenz 3,1 Proz. Bei der Gesamteinwanderung dagegen gab es 23,3 Proz. der verheirateten Männer und 9,6 Proz. der verheirateten Frauen. Die Differenz 13,7 Proz. Noch größer ist der Unterschied bei den Italie- 
nern: $30,7-9,8=20,7$ Proz. Das heißt: bei den Juden kommen viel mehr verheiratete Männer $m$ it ihren Frauen an, als bei der Gesamteinwanderung und den Italienern. Die Judenwanderung ist eine Familienwanderung.

Die Zahl der Witwer im Alter von 14-44 Jahren ist bei den Juden, der Gesamteinwanderung und den Italienern fast dieselbe, jedoch übersteigt die Zahl der jüdischen Witwer im Alter über 45 Jahre (in Prozent: 0,4) die Zahl der Witwer in der Gesamteinwanderung (in Prozent: 0,3) und bei den Italienern (in Prozent: 0,2). Die jüdischen Witwen sind jedoch in beiden Altersklassen stärker vertreten als unter den übrigen Einwanderern. Auch die Älteren, die verwitweten Väter und Mütter, ziehen mit den jüngeren in die neue Heimat herüber.

Auf den starken Familienzusammenhang zwischen den schon früher Ausgewanderten und den Nachziehenden. oder überhaupt zwisclien der alten und neuen Heimat könner wir aus der Zahl derjenigen Auswanderer schließen, die ihre Fahrt nicht selbst bezahlt haben, sondern die Reisekosten von ihren Verwandten bekommen haben. Die Zahl solcher Auswanderer ist bei den Juden recht groß: in den letzten 3 Jahren (1912-14) betrug sie $211765, \mathrm{~d} . \mathrm{h}$. 66,1 Proz. (Tabelle XVI.) Selbst haben die Fahrt unter den Juden nur 105942 Auswanderer, d. h. 33,4 Proz., bezahlt; weder selbst noch von Verwandten wurde die Reise von 1269 Personen, d. h. 0,3 Proz., bezahlt. Dementsprechend gingen zu Verwandten 94 Proz, aller Auswanderer dieser drei Jahre! $Z u$ Freunden gingen 4,0 Proz., und weder zu Freunden noch zu Verwandten nur 1,9 Proz. Diese Zahlen sprechen recht deutlich von dem bewunderungswürdigen jüdischen Gemeinschaftsgefühl.

Unter allen Nationalitälen, die in den Jahren 1912 bis 14 nach Amerika einwanderten, wurde bei den Juden die Reise von den Verwandten am häufigsten bezahlt: sie stehen in dieser Beziehung mit ihren 66,1 Proz. an der Spitze; es folgen dann Litauer mit 49,5 Proz., Deutsche mit 39,9 Proz., Franzosen mit 37,8 Proz., Engländer mit 36,9 Proz. usw. (Tabelle XVII.) 


\section{$-26-$ \\ Tabelle XVI \\ Auswanderer auf eigene und fremde Rechnung}

\begin{tabular}{|c|c|c|c|c|c|c|}
\hline Jahr & $\begin{array}{l}\text { Die Reise } \\
\text { Staate } \\
\text { selbst } \\
\text { abculut } \mid \begin{array}{c}\text { in } \\
0_{0}\end{array}\end{array}$ & $\begin{array}{l}\text { in dic Verei } \\
n \text { wurde heza } \\
\text { von } \\
\text { Verwandten } \\
\text { absolul in } \\
0_{0}\end{array}$ & $\begin{array}{l}\text { einigten } \\
\text { ahit } \\
\text { weder } \\
\text { selbst noch } \\
\text { von V'T- } \\
\text { wandten } \\
\text { ab- in } \\
\text { solut a }\end{array}$ & \begin{tabular}{|c} 
zul \\
Verwandten \\
abrolut in
\end{tabular} & $\begin{array}{l}\text { in } \\
\text { Frtulen } \\
\text { absolut }{ }_{\text {in }}\end{array}$ & $\begin{array}{l}\text { weder zu } \\
\text { Verwandten } \\
\text { noch zu } \\
\text { Freunden } \\
\text { ab- in } \\
\text { solut }{ }^{\circ} \text { " }\end{array}$ \\
\hline $1: 111-19112$ & 35,930319 & $5.1539467,6$ & $24+0,3$ & $T(6)(1 ; 3), 91,3$ & $301063 \quad 3,7$ & $1506 i, 1,8$ \\
\hline $191: 3-19133$ & $36+23 \quad 35,9$ & $64+00 \quad 63,6$ & $50-0,5$ & $945011,48,3$ & $4437: 4,7$ & $16(0) 2,1,6$ \\
\hline $1919-1914$ & 4174732,4 & $92+266 \pi, 5$ & 4780,2 & 13015494,3 & 300993,6 & 25582,1 \\
\hline
\end{tabular}

Auch was die Zahl derjenigen Einwanderer, die zu den Verwandten gingen, anbetrifft, stehen die Juden an der Spitze aller anderen Nationalitäten; im übrigen ist es bezeichnend, daß auch bei den anderen Nationalitäten die Zahl der Einwanderer, die zu den Verwanditen gingen, recht groß ist, am kleinsten bei den Engländern: 94505 Einwanderer, d. h, 60,1 Proz.

\section{Finanzielle Lage der jüdischen Einwanderer}

Omnia sua secum portat - das antike Wort ist auf den jüdischen Wanderer ohne weiteres anzuwenden. Wie ist seine finanzielle Lage? Tab. XVIII gibt dariber sehr reiche Aufschlüsse. Es kamen auf den Kopf der jüdischen Einwanderer zwischen 7,3 und 24,4 Dollars, während bei der Gesamteinwanderung jeder Einwanderer zwischen 15 und 36,2 Dollars mitbrachte. Die Verbesserung, die in clen vorletzten zwei Jahren eintrat und bei der Gesamtbevölkerung auch weiter anhielt, ist bei den Juden im Jahre 1913-14 nicht mehr festzusiellen. Die Zahl der jüdischen Geldträger ist überhaupt niedriger als bei der Gesamteinwanderung, da die Juden am meisten Kinder unter 14 Jahren und Frauen in ihren Reihen aufweisen. Jedoch stehen auch 


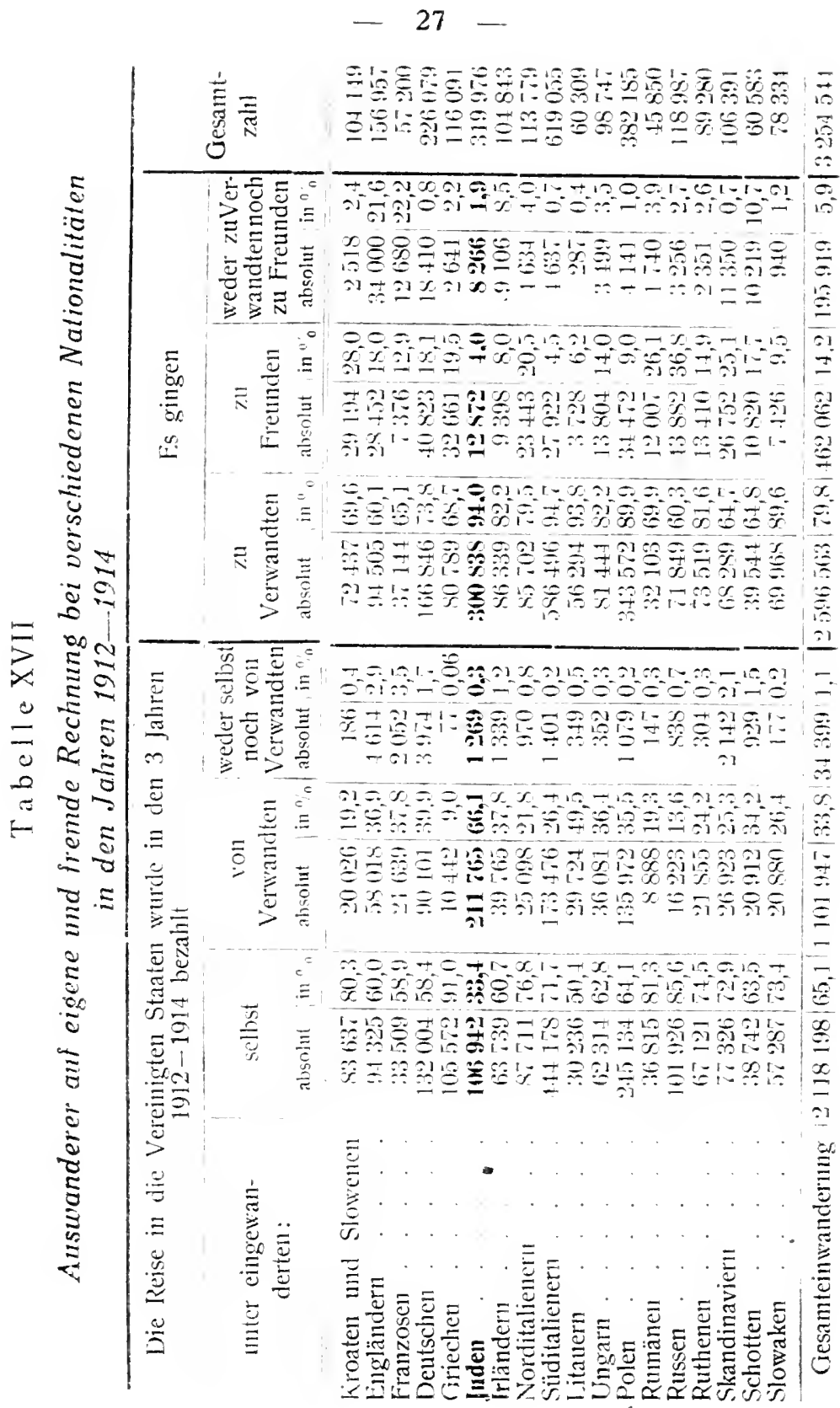


T a belle XVIII

Finanzielle Lage der jüdischen Einwanderer

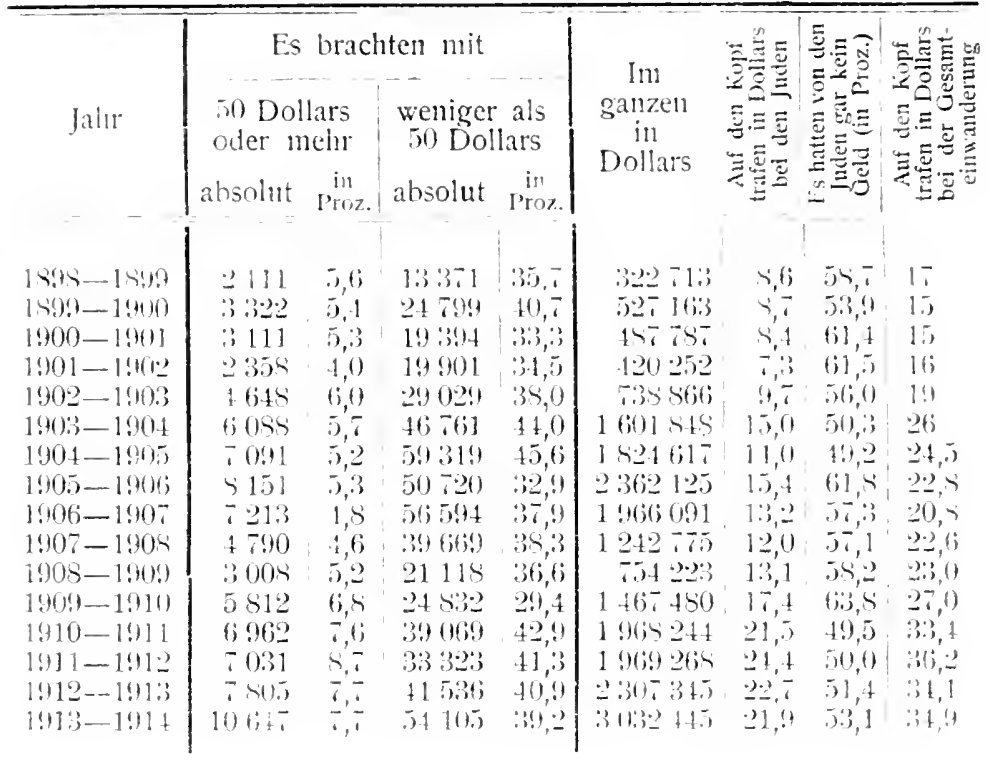

unter Heranziehung dieses Umsiandes die Juden hinter der Gesamteinwanderung zurück, obwohl sie dann nicht mehr die ärmste Einwanderungsbevölkerung bilden. Das besprochene Moment wird allerdings zum Teil dadurch aufgewogen, daß die von den Juden mitgebrachte Geldsumme die Grundlage ihrer dauernden Niederlassung bilden soll, während die übrigen Einwanderer, die nur auf beschränkte Zeit nach Amerika kommen, und dazu nocl meist ohne Familie, weniger Ausgaben zu machen haben. Die Juden müssen mit dem mitgebrachten Geld im Durchschnitt mehr Bedürfnisse decken als die übrigen Einwanderer.

Die finanzielle Lage der jüdischen Einwanderer läßt mithin noch viel zu wünschen übrig. Die Zahl derjenigen, die 50 oder mehr Dollars mitbringen, beträgt zwischen 4 und 7,5 Proz. Nur im Jahre 1911-12 beträgt der Prozentsatz der "Reichen" 8,7 Proz. Auch die Zahl der jüdischen Einwanderer, die gar kein Geld hatten, ist recht groß. Es 
 \\ Tabelle XIX}

Zahl der zurückgewiesenen Juden in den Jahren 1912-1914

\begin{tabular}{|c|c|c|c|c|c|c|c|c|c|}
\hline \multirow{3}{*}{ Jahr } & \multirow{2}{*}{\multicolumn{2}{|c|}{$\begin{array}{l}\text { Gesant- } \\
\text { zalil der } \\
\text { Zurüickge- } \\
\text { wiesenen }\end{array}$}} & \multicolumn{7}{|c|}{ Grund der Zurückweisung } \\
\hline & & & \multicolumn{2}{|c|}{ Trachoma } & $\begin{array}{l}\text { Könnten der } \\
\text { öffentlichen } \\
\text { Armen- } \\
\text { pflege zur } \\
\text { Last fallen }\end{array}$ & \multicolumn{2}{|c|}{$\begin{array}{l}\text { Arztliches Attest } \\
\text { iiber einen körper- } \\
\text { lichen oder geisti- } \\
\text { gen Defeki, der die } \\
\text { Fähigkeit, seinen } \\
\text { Lebensunterhalt } \\
\text { zu verdienen, be- } \\
\text { einflussen könnte }\end{array}$} & \multicolumn{2}{|c|}{$\begin{array}{l}\text { Ver- } \\
\text { schiedene } \\
\text { Gründe }\end{array}$} \\
\hline & absolut & & $\begin{array}{l}\text { ab- } \\
\text { solut }\end{array}$ & $i_{11}$ & \begin{tabular}{l|l} 
absolut & in \\
\end{tabular} & rbsolut & in 0 & $\begin{array}{l}\text { ah- } \\
\text { soluit }\end{array}$ & $\begin{array}{l}\text { in } \\
{ }_{0}^{0}\end{array}$ \\
\hline $1911-1912$ & $106 \cdot 1$ & 1,3 & 162 & 15,3 & $455+2,6$ & 2.66 & 23,1 & 201 & 18,8 \\
\hline $1912-1913$ & 1224 & 1,2 & 132 & $10, \overline{7}$ & $417: 30,0$ & 401 & 32,5 & 244 & 19,2 \\
\hline $1913-1914$ & 2506 & 1,8 & 222 & 8,8 & $1055 \quad 42,2$ & 838 & 33,4 & 391 & 35,6 \\
\hline $1912-1914$ & 4.994 & 1,5 & 516 & 10,7 & 195740,9 & 1485 & 30,9 & 836 & 17,5 \\
\hline
\end{tabular}

Tabelle XX

Zahl der zurückgewiesenen Einwanderer in der italienischen, jüdischen und der Gesamteinwanderung in den Jahren 1912-1914

\begin{tabular}{|c|c|c|c|c|c|c|c|c|c|c|}
\hline \multirow{3}{*}{ Nationalität } & \multirow{2}{*}{\multicolumn{2}{|c|}{$\begin{array}{l}\text { Gesanit- } \\
\text { zahl der } \\
\text { Zurïck- } \\
\text { gewiesenen }\end{array}$}} & \multicolumn{8}{|c|}{ Grund der Znrückweis $11 n g$} \\
\hline & & & Trach & oma & $\begin{array}{c}\text { Könute } \\
\text { öffentli } \\
\text { Arme } \\
\text { pflege } \\
\text { Last ia }\end{array}$ & $\begin{array}{l}\text { nder } \\
\text { chen } \\
\text { n- } \\
\text { zur } \\
\text { llen }\end{array}$ & $\begin{array}{l}\text { Ärztlich } \\
\text { über eine } \\
\text { lichen od } \\
\text { gen Defe } \\
\text { Fähigke } \\
\text { Lebens } \\
\text { zul verdi } \\
\text { einflusse }\end{array}$ & $\begin{array}{l}\text { Attest } \\
\text { korper- } \\
\text { geisti- } \\
\text { der die } \\
\text { seinen } \\
\text { erhalt } \\
\text { n, be- } \\
\text { könnte }\end{array}$ & $\begin{array}{l}\text { Ve } \\
\text { schied } \\
\text { Griit }\end{array}$ & de \\
\hline & absolut & $\begin{array}{l}\text { in } \\
\% \% \\
\end{array}$ & $\begin{array}{c}\text { ab- } \\
\text { solut } \\
\end{array}$ & $\begin{array}{l}\text { in } \\
0 \\
0\end{array}$ & absolut & in & absolut & in $0_{0}^{\prime}$ & absolut & $\begin{array}{l}\text { in } \\
0 \% \\
0\end{array}$ \\
\hline Italiener. . & 13218 & 1,8 & 197 & 7,2 & $4466 ;$ & 34,2 & 4174 & 39,2 & 3651 & 28,2 \\
\hline Juden. . & +794 & $1, \mathrm{~S}$ & 516 & 10,7 & 195 & 40,9 & 145.5 & 30,9 & $\$ 36$ & 17,5 \\
\hline $\begin{array}{l}\text { Gesamteiu- } \\
\text { wanderung }\end{array}$ & 69036 & 2,1 & 5933 & 8,6 & 31838 & 46,1 & 13033 & 18,8 & 18232 & 26,1 \\
\hline
\end{tabular}

gab deren mehr als die Hälfte aller jüdischen Einwanderer; ziehen wir davon den Prozentsatz der Kinder ab, so bleiben doch 25-30 Proz. der jüdischen Einwanderer übrig, die völlig mittellos sich in der neuen Heimat einfanden. 
Tabelle XXI

Bildungsverhältnisse der jüdischen Einwanderer

\begin{tabular}{|c|c|c|c|c|c|}
\hline \multirow[b]{2}{*}{ Jahr } & \multicolumn{3}{|c|}{ Analphabeten vom 14. Jalıre ab } & 幽总羿 & $\overline{\bar{\Xi}}=\bar{\Xi}$ \\
\hline & $\begin{array}{c}\text { Können } \\
\text { lesen, aber } \\
\text { nicht schreiben } \\
\text { Männlich / W'ciblich }\end{array}$ & $\begin{array}{c}\text { Kömnen } \\
\text { weder lesen } \\
\text { noch schreiben } \\
\text { Nännlich Weiblich }\end{array}$ & 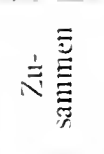 & 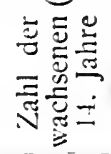 & 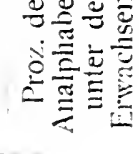 \\
\hline $1911 \ldots 19112$ & 393 & !) +498 & $15+2 \mathrm{~s}$ & dio 504 & $2.2,::$ \\
\hline $1912-1913$ & $2 \cdot 2 \cdot 3$ & $65 t i 3,10099$ & $11 ; 950$ & 78452 & $\stackrel{-1,5}{ }$ \\
\hline $1913-1914$ & $20 \div$ & 13591 & 21490 & 107938 & $1 ! 1,4$ \\
\hline $1912-1911$ & (iti 251 & 1981:3 :3:3 & 3:34. & $2+73994$ & $\because$ \\
\hline
\end{tabular}

Dementsprechend ist auch die Zahi der von der Landung $Z$ urü ckgewiesenen bei den Juden zwar beträchtlich (in den letzten 3 Jahren 1,8 Proz.), jedoch kleiner als bei der Gesamteinwanderung (2,1 Froz.) und nicht größer als bei den Italienern (1,8 Proz.) (Tabellen XIX und $\mathrm{XX}$ ). Die meisten Juden wurden deshaib zurückgewiesen, weil die Annahme berechtigt war, daß sie der öffentlichen Armenpflege zur Last fallen könnten (40,9 Proz. aller Zurückgewiesenen). Bei der recht großen Anzahl von Juden lag ein ärztliches Attest über einen körperlichen oder geistigen Defekt vor, der die Fähigkeit, seinen Lebensunterhalt zu verdienen, ungünstig beeinflussen könnte (in den Jahren 1912-14 durchschnittlich 30,9 Proz). Bei den Italienern 39,2 Proz., bei der Gesamteinwanderung 18,8 Proz. Infolge von Trachomakrankheit wurden bei den Juden in den Jahren 1912-14 10,7 Proz. zurückgewiesen, bei den Italienern 7,2, bei der Gesamteinwanderung 8,6 Proz.

\section{Bildungsverhältnisse der jüdischen Einwanderer}

Über die Bildungsverhältnisse unter den jüdischen und den Einwanderern der andern Nationalitäten in den letzten 3 Jahren unterrichten Tabellen XXI und XXII. Dem- 


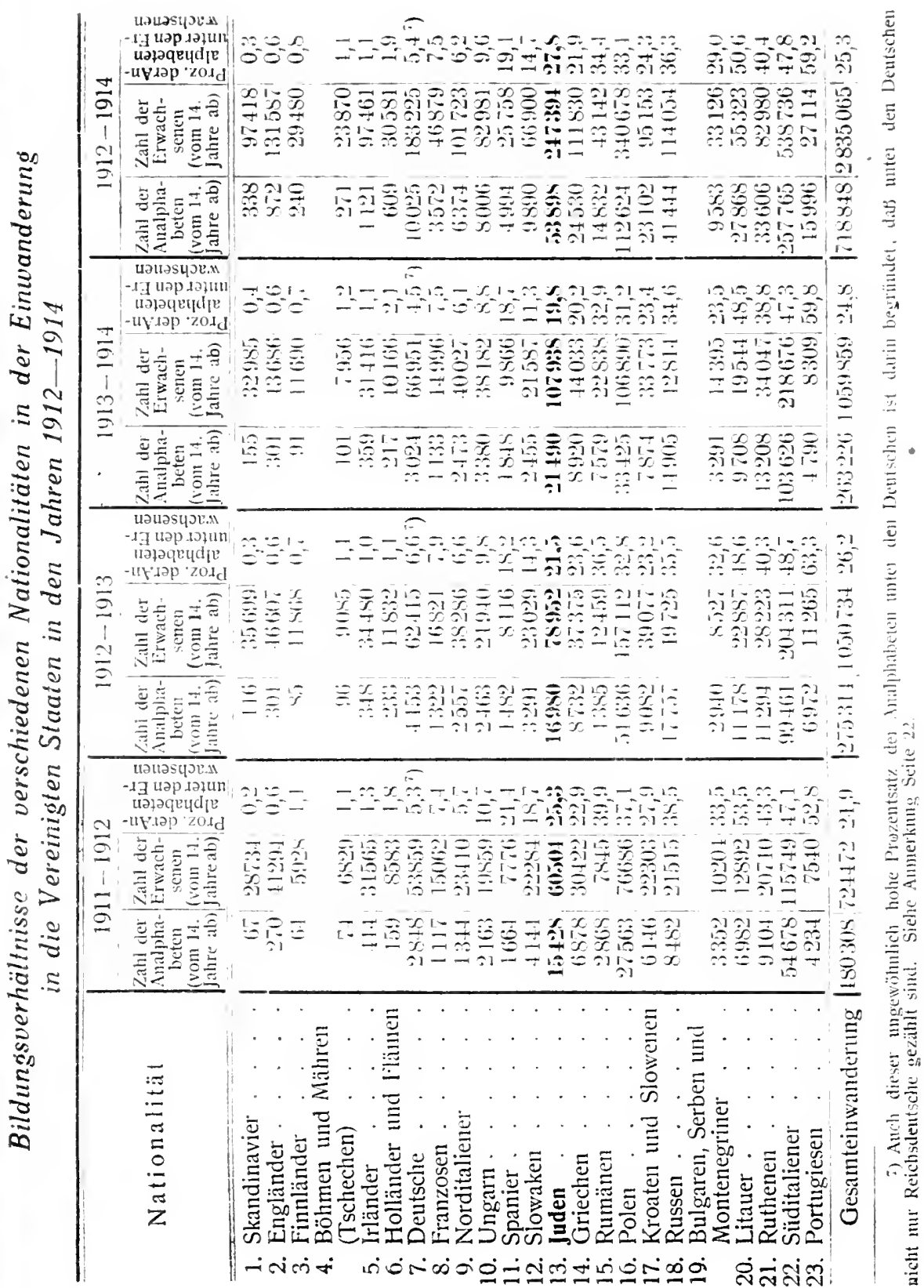




\section{- $32-$}

nach stehen die Juden, was die Zahl der Analphabeten unter den Erwachsenen (vom 14. Jahre ab) anbetrifft, an dreizehnter Stelle; es konnten unter ihnen lesen aber nicht schreiben 897 Personen (in den letzten 3 Jahren 1912-14), und weder lesen noch schreiben 53001 Personen, zusammen 53898 Personen, d. h. 27,8 Proz. aller Erwachsenen. Am meisten Analphabeten befanden sich unter den Portugiesen (59,2 Proz.), dann unter den Litauern (50.6 Proz.). 



\section{PLEASE DO NOT REMOVE CARDS OR SLIPS FROM THIS POCKET}

$$
\text { UNIVERSITY OF TORONTO LIBRARY }
$$


